\title{
Digital Archives for Nuclear Emulsion Data
}

\author{
Koichi Kodama ${ }^{1, *}$, Takehiro Kamiya ${ }^{1}$, Masakatsu Ichimura $^{2}$, and Mitsuhiro Nakamura ${ }^{3}$ \\ ${ }^{1}$ Aichi University of Education, Kariya, 448-8524 Japan \\ ${ }^{2}$ Faculty of Science and Technology, Hirosaki University, Hirosaki, 036-8561 Japan \\ ${ }^{3}$ Nagoya University, Nagoya, 464-8602 Japan
}

\begin{abstract}
Digital archives for nuclear emulsion data of past experiments, such as in cosmic-ray and accelerator physics, is being studied and prepared. Significant progress of HTS, which is an automatic read-out system for tracks recorded in emulsion, is achieving a read-out speed of about $1 \mathrm{~m}^{2} /$ hour and opens a possibility to read all tracks recorded in emulsion of past experiments. Current status of our first trial with RUNJOB emulsion plates is reported. Till now, the top-most 10 plates had been scanned by HTS and preliminary data is presented.
\end{abstract}

\section{Introduction}

Trajectories or tracks of charged particles are recorded in nuclear emulsion as series of silver grains as shown in Fig.1. The size of each grain is $\sim 1$ micron and those tracks can be easily observed under optical microscope views. Automatic read-out systems for those tracks in emulsion had been playing an important role in elementary particle physics, for example, contributing in the first direct observation of tau neutrino charged current interactions in the DONUT experiment [1], and in the discovery of tau neutrino appearance in the CNGS neutrino beam with the OPERA experiment [2]. A most recent system called HTS [3] is achieving read-out speeds of $\sim 1 \mathrm{~m}^{2}$ /hour making it possible to process $2000 \mathrm{~m}^{2}$ of emulsion surface in one year. This value is similar to full emulsion surface of past experiments in accelerator and in cosmic-ray physics, such as $\sim 100 \mathrm{~m}^{2}$ for DONUT, $\sim 120 \mathrm{~m}^{2}$ for RUNJOB and $\sim 900 \mathrm{~m}^{2}$ for JACEE.

In such past experiments, analysis or read-out speed of emulsion was limited and thus their analysis had to concentrate on events or phenomena of their major physics aims, with the help of supporting detectors such as scintillating fiber trackers, muon detectors and x-ray films.

Most events or phenomena recorded but not matching their major physics aims remained as not read-out nor analysed. As it is not so easy to have such events or phenomena recorded in emulsion again by performing new experiments, then it should be interesting and valuable to read full surface of such past emulsions and perform new physics analysis from our current physics knowledge. For such activities, a kind of archive for emulsion data, which is open to those who are interested, would be a suitable scheme. As a first such trial, one chamber in RUNJOB balloon experiment is being processed and the status of the trial is reported hereafter.

\footnotetext{
*e-mail: kkodama@auecc.aichi-edu.ac.jp
}

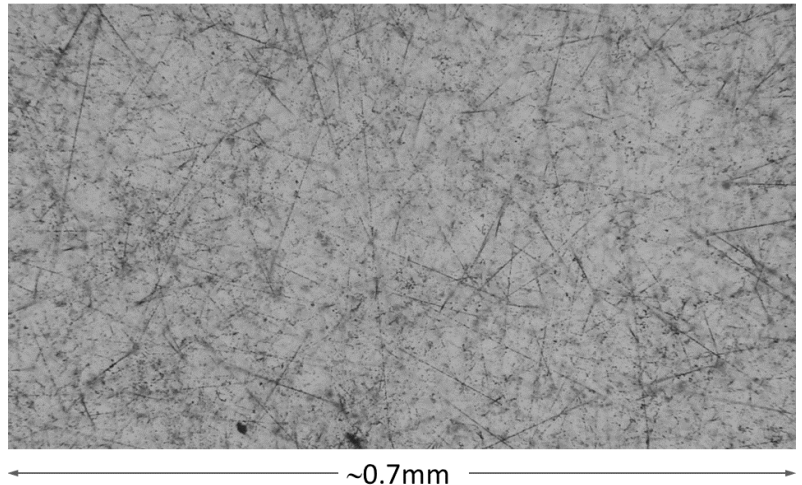

Fig 1. Microscope image of tracks of charged particles recorded in an emulsion plate of the RUNJOB experiment.

\section{The RUNJOB Experiment}

RUNJOB (RUssia Nippon JOint Balloon program) is a balloon experiment aiming to measure the chemical composition and energy spectra of the primary cosmic rays [4]. There were 4 balloon campaigns from 1995 to 1999 and a total of 10 balloons were successfully recovered. Each balloon flight was for $\sim 140$ hours flight time at a mean altitude of $\sim 10 \mathrm{~g} / \mathrm{cm}^{3}$ or $30 \sim 35 \mathrm{~km}$. One of two chambers in the 1997 flight is being processed by HTS. The chamber has a cross section of $50 \mathrm{~cm} \times 40 \mathrm{~cm}$. An emulsion plate has two $60 \mu \mathrm{m}$ thick emulsion layers poured on both sides of a plastic base, whose thicknesses are $500 \mu \mathrm{m}$ or $800 \mu \mathrm{m}$. The chamber structure is composed of 5 modules as schematically shown in Fig.2. Emulsion plates are interleaved with materials selected, such as stainless plates in the target module, $x$-ray films and lead plates in the calorimeter module, according to their purposes. 


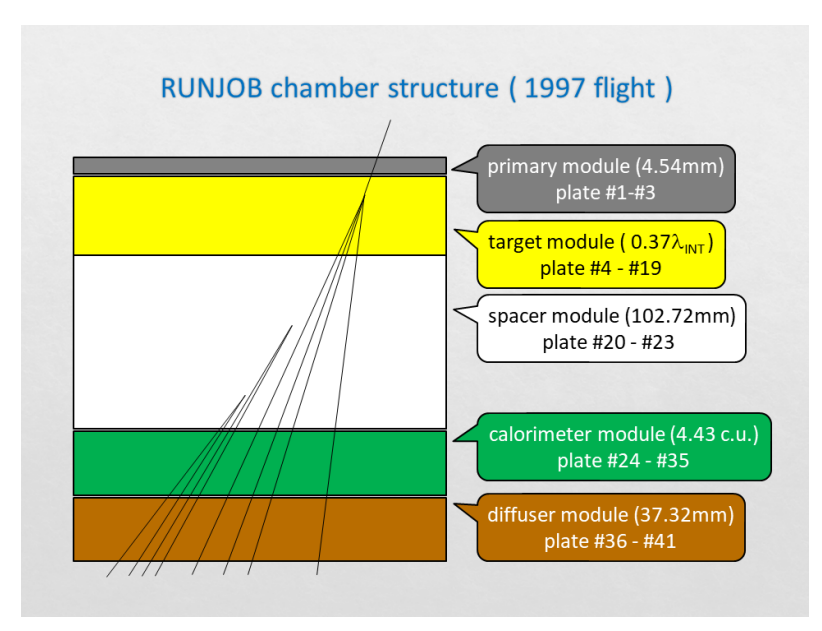

Fig 2. Structure of a RUNJOB chamber for the 1997 flight.

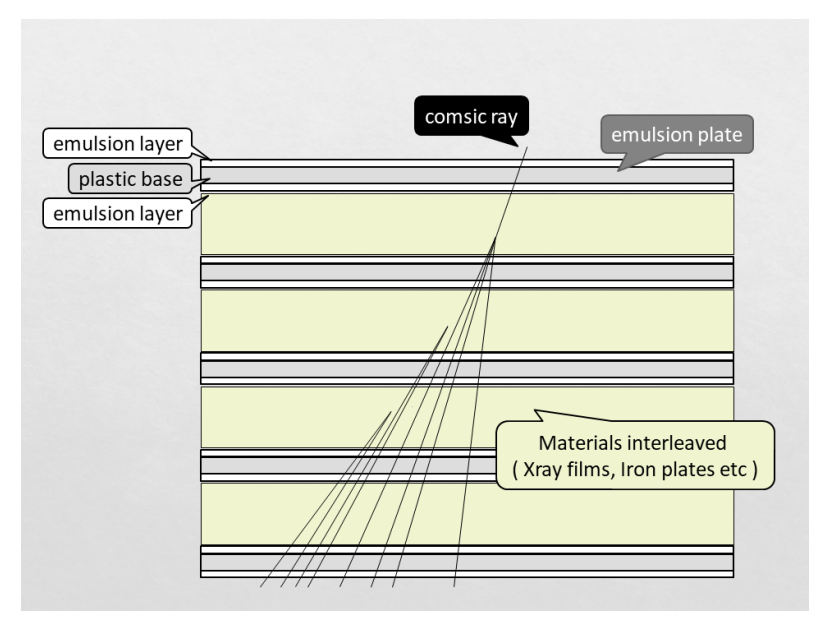

Fig 3. A schematic picture of a cosmic ray interaction occurring and recorded in an emulsion chamber.

\section{Emulsion Data Process}

\subsection{Track Read-out by HTS}

Before reading-out emulsion tracks by HTS scan, some treatments have to be applied. The size of a RUNJOB emulsion plate is $50 \mathrm{~cm} \times 40 \mathrm{~cm}$, which is too large for the HTS stage, and it had to be cut into two pieces (two halfplates) of size $25 \mathrm{~cm} \times 40 \mathrm{~cm}$. Then they have to be swollen to expand their emulsion layers thickness from $\sim 30 \mu \mathrm{m}$ to $\geq 60 \mu \mathrm{m}$, so that HTS could efficiently read-out emulsion tracks in them. An emulsion plate has two emulsion layers on both sides of a plastic base and HTS scans each layer separately. Track segments in those layers are called "micro-tracks". Each RUNJOB half-plate is scanned divided into 9 zones, since one HTS scan is limited up to $13 \mathrm{~cm} \times 9 \mathrm{~cm}$, So far, the top-most 10 plates of the chamber had been scanned with angular acceptance $\tan \theta \leq 1.4$.

\subsection{Emulsion Data Reconstruction}

Fig.3 schematically shows an interaction induced by a charged particle entered from upside of the emulsion chamber. Track information that we can obtain for this

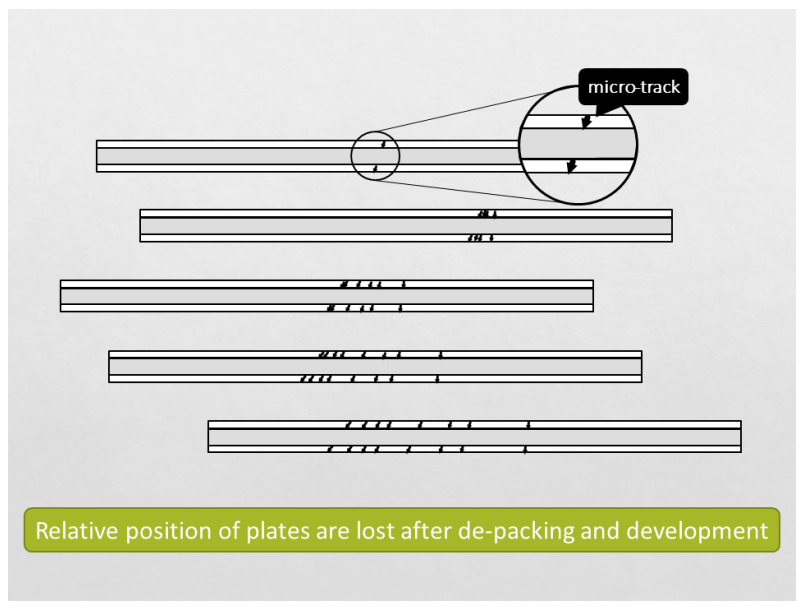

Fig 4. A schematic picture of micro-tracks related to the cosmic ray interaction in Fig.3. Precise relative position among emulsion plates are lost after they were de-packed and developed.

event as a result of HTS scan looks as shown in Fig.4, since tracks of charged particles are recorded only in emulsion layers and relative positions among emulsion plates are lost after they were de-packed and developed. It means that connections among micro-tracks (i.e. information of which micro-track is to be attached to which charged particle) is more or less lost. Thus, simply speaking, the purpose of emulsion data processing is to reconstruct Fig.3 from Fig.4.

The first step is to reconstruct connections between micro-tracks in two emulsion layers of a plate. Distortion and shrinkage of emulsion layers are evaluated by searching for a set of those parameters that maximizes the number of connected micro-tracks within the tolerance defined by their position and angle accuracy. A connection between those micro-tracks is called a "base-track". To keep high connection efficiency, all possible connections between micro-tracks are kept as base-tracks and thus one micro-track could be connected to multiple micro-tracks.

After making base-tracks, the relative position between two emulsion plates have to be reconstructed. This is also done by searching for relative position that maximizes the number of connected base-tracks. During such an alignment process of the RUNJOB chamber, some of the relative positions between two emulsion plates are found moving or slipping. A typical example of such slipping is shown in Fig.5. This would be due to temperature change and/or mechanical vibrations while in their balloon flight.

Using obtained relative positions between two plates, base-tracks on different plates are connected to form what is called "linklets". The slipping observed in the above alignment process must be taken into account properly in forming those linklets. To keep high connection efficiency, all possible connections within the tolerance are kept and thus one base-track could be connected to multiple basetracks. To recover the inefficiency of base-tracks, linklets between non-consecutive plates are also used. Finally, connections among linklets in different plates are traced 


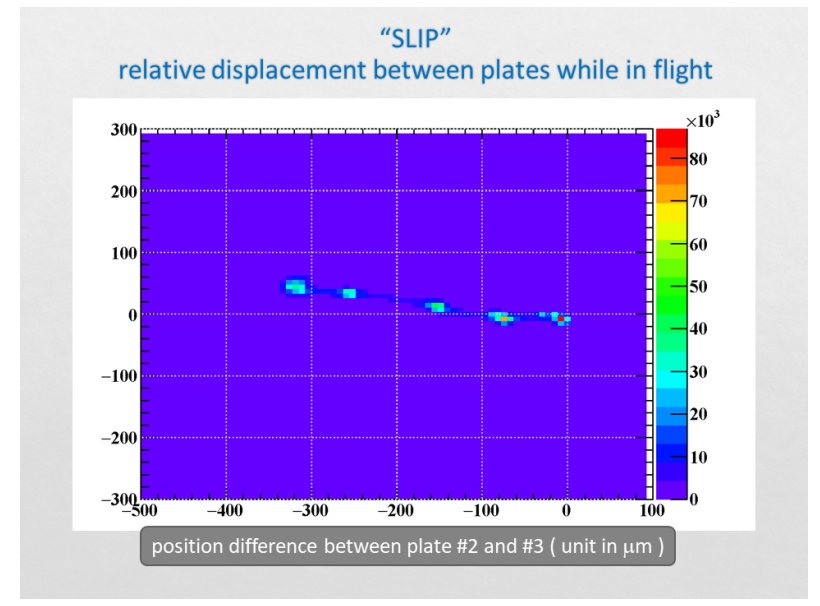

Fig 5. An example of "slipping". Relative position between emulsion plates had been moving, most probably while the chamber was in its balloon flight.

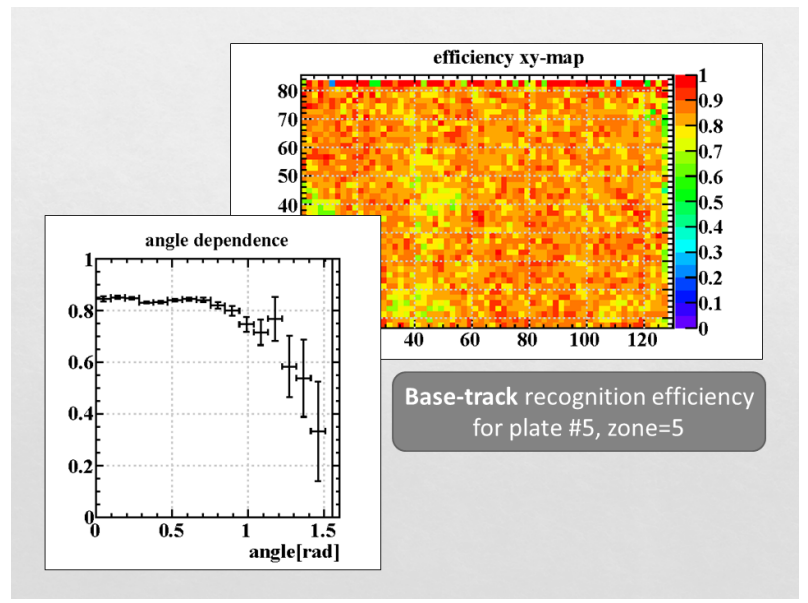

Fig 6. An example of base-track recognition efficiency for plate $\# 5$ in zone $=5$, which is $\sim 0.85$ for $\tan \theta \leq 1.0$. It should be noted that it depends on plate quality and is not quite uniform.

to reconstruct charged particles recorded in the chamber. Those connections among linklets are called "chains".

\subsection{Track Recognition Efficiency}

The recognition efficiencies of base-tracks in RUNJOB emulsion plates are evaluated by simply counting the number of missing base-tracks in a chain, using chains that penetrate all scanned emulsion plates and are isolated from other chains. A result for plate $\# 5$, zone $=5$ is shown in Fig.6. The recognition efficiency was $\sim 0.82$ for $\tan \theta \leq 1.0$, that would be acceptable for physics analysis. It must be noted that the efficiency varies depending on plate quality, which is not quite uniform.

\section{Analysis Examples}

\subsection{Vertex Search}

Vertices such as due to hadronic interactions could be selected using linklets. As a first step, all pairs of linklets,

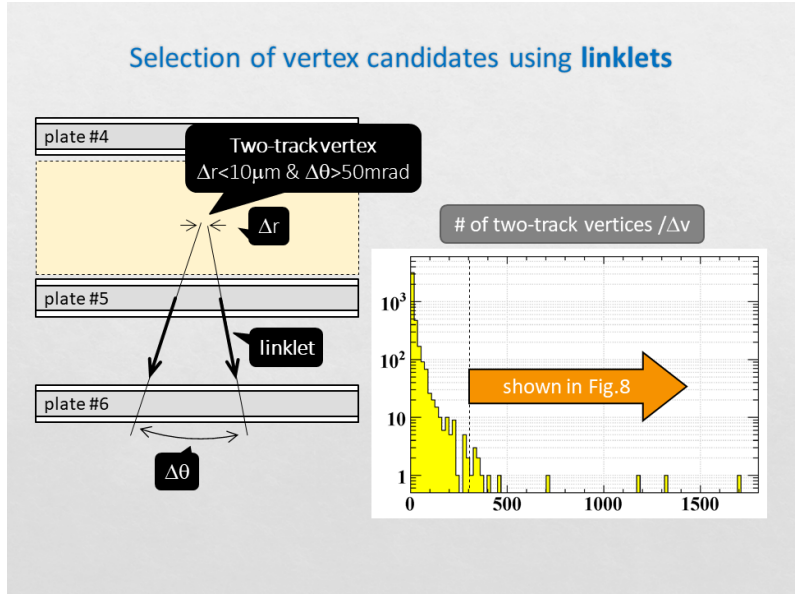

Fig 7. A method to search for vertices using linklets. Vertices in a region just upstream of plate \#5, where a stainless plate is placed, are searched.

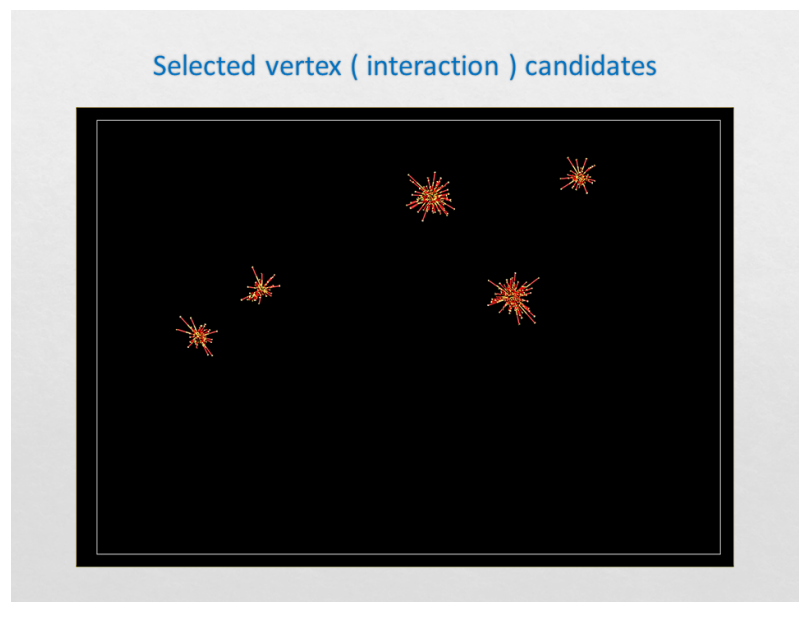

Fig 8. 3D display of selected vertices using linklets between plate $\# 5$ and $\# 6$ in zone $=5(13 \mathrm{~cm} \times 9 \mathrm{~cm})$. The view is looking into the emulsion surface.

each converge to a point called a "two-track vertex", are picked up within the tolerance in a region where vertices are to be searched. Then, a 3D histogram of positions of those two-track vertices are made to select histogram bins, in which two-track vertices are concentrated.

As an example, this method is applied to all linkets between plate \#5 and \#6 in one zone to find vertices in stainless plate placed just upstream of plate \#5 (see Fig.7). All pairs of linklets having closest distance $\Delta \mathrm{r} \leq 10 \mu \mathrm{m}$ and relative angle difference $\Delta \theta \geq 50 \mathrm{mrad}$ are selected. The $3 \mathrm{D}$ histogram of positions of selected two-track vertices are made with a bin size $20 \mu \mathrm{m} \times 20 \mu \mathrm{m}$ in a plane parallel to emulsion plates and $40 \mu \mathrm{m}$ in the vertical. A histogram of the number of two-track vertices in each $3 \mathrm{D}$ histogram bins is shown in Fig.7. Then linklets which belongs to twotrack vertices in 3D histogram bins having $\geq 300$ entries are shown in Fig.8. 
Selection of shower candidates using group of chains

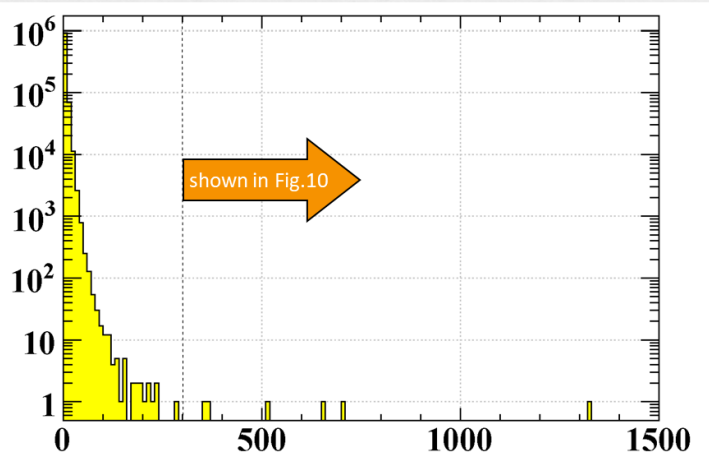

Fig 9. The histogram shows how many base-tracks each group of chains has. Those group of chains having $\geq 300$ base-tracks are selected and those base-tracks are shown in Fig.10.

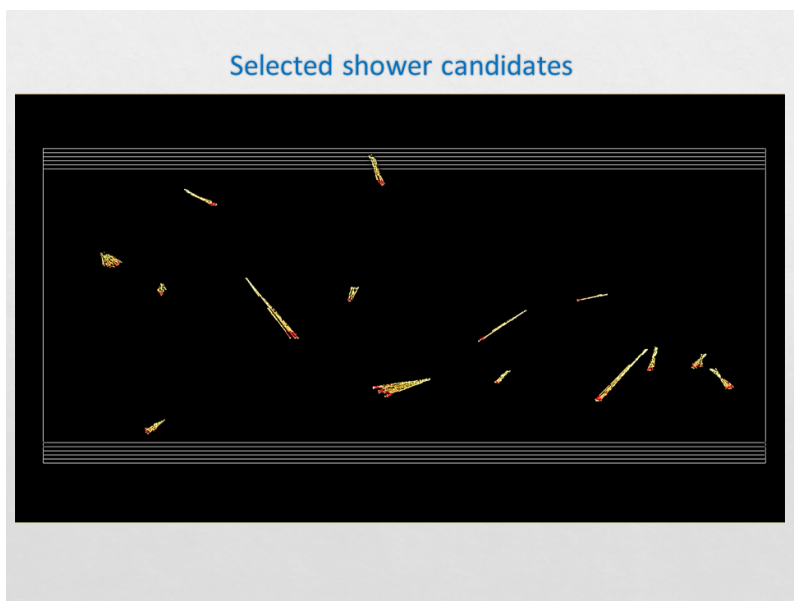

Fig 10. 3D display of selected shower candidates in plates from $\# 5$ to $\# 10$ in zone $=5(13 \mathrm{~cm} \times 9 \mathrm{~cm})$. Since they are in the target module, they are more likely to be hadronic showers or interactions.

\subsection{Shower Search}

Shower topology could be better selected using chains. For example, in electro-magnetic showers, many tracks having similar angles are populated in a small region on each emulsion plate. In such circumstance, connection of base-tracks between two plates are more likely to be multiple to multiple due to insufficient angle accuracy of base-tracks. Then there are most likely to be many chains having same base-tracks in common, and those chains are to be grouped. Shower topologies could then be selected by choosing groups of chains having many chains and/or base-tracks in their members.

As an example, this method is applied for chains in plates from \#5 to \#10 ( i.e. in the target module of the chamber ). A distribution of the number of base-tracks in each group of chains is shown in Fig.9 and those groups having $\geq 200$ base-tracks are then selected and shown in Fig. 10 .

\section{Summary}

Recent developments of HTS ( an automatic read-out system for emulsion tracks ) are achieving a speed of $\sim 1 \mathrm{~m}^{2}$ /hour and it is being possible to process $2000 \mathrm{~m}^{2}$ in one year. This allows us to read the full surface of emulsion plates of past experiments in accelerator and in cosmic-ray physics. Since most of the events or phenomena recorded in those emulsion plates are not read-out and remain un-used, and it is also not so easy to have them recorded in emulsion again by new experiments, it should be interesting and valuable to read them all to construct a digital archive of those emulsion data. As a first trial of such archives, one chamber of RUNJOB balloon experiment is being processed. Up to now, top-most 10 emulsion plates had been read-out by HTS. The recognition efficiency of base-tracks is evaluated to be $\sim 0.85$ for $\tan \theta \leq 1.0$, that would be acceptable for physics analysis. Relative slipping between emulsion plates was observed but could be treated properly without serious problem. As an analysis example, searches for vertices and showers are shown using a subset of obtained data.

After the conference, big efforts are being devoted to an HTS scan of the remaining emulsion plates of the chamber and will be completed in 2018 .

\section{References}

[1] DONUT Collab., Phys.Rev. D78, 052002 (2008)

[2] OPERA Collab., Phys.Rev.Lett. 115, 112802 (2015)

[3] M.Yoshimoto, T.Nakano, R.Komatani, H.Kawahara, PTEP 2017, 103H01 (2017)

[4] RUNJOB Collab., Astropart. Phys. 16, 13-46 (2001) 